University of New Hampshire

University of New Hampshire Scholars' Repository

Space Science Center

Institute for the Study of Earth, Oceans, and

Space (EOS)

1994

\title{
Observations of the 1991 June 11 solar flare with COMPTEL
}

\author{
G Rank \\ Max-Planck-Institut für extraterrestrische Physik \\ R Diehl \\ Max-Planck-Institut für extraterrestriche \\ G G. Lichti \\ Max-Planck-Institut für extraterrestrische Physik \\ V Schonfelder \\ Max-Planck-Institut für extraterrestrische Physik \\ M Varendorff \\ Max-Planck-Institut für extraterrestrische Physik
}

See next page for additional authors

Follow this and additional works at: https://scholars.unh.edu/ssc

Part of the Astrophysics and Astronomy Commons

\section{Recommended Citation}

Observations of the 1991 June 11 solar flare with COMPTEL Rank, G. and Diehl, R. and Lichti, G. G. and Schönfelder, V. and Varendorff, M. and Swanenburg, B. N. and van Dijk, R. and Forrest, D. and Macri, J. and McDonnell, M. and Loomis, M. and Ryan, J. and Bennett, K. and Winkler, C., AIP Conference Proceedings, 294, 100-105 (1994), DOl:http://dx.doi.org/10.1063/1.45207

This Conference Proceeding is brought to you for free and open access by the Institute for the Study of Earth, Oceans, and Space (EOS) at University of New Hampshire Scholars' Repository. It has been accepted for inclusion in Space Science Center by an authorized administrator of University of New Hampshire Scholars' Repository. For more information, please contact Scholarly.Communication@unh.edu. 


\section{Authors}

G Rank, R Diehl, G G. Lichti, V Schonfelder, M Varendorff, B Swanenburg, R VanDijk, D J. Forrest, John R. Macri, Mark L. McConnell, M Loomis, James M. Ryan, K Bennett, and C Winkler 


\section{AIP | Proceedings}

\section{Observations of the 1991 June 11 solar flare with COMPTEL}

G. Rank, R. Diehl, G. G. Lichti, V. Schönfelder, M. Varendorff, B. N. Swanenburg,

R. van Dijk, D. Forrest, J. Macri, M. McDonnell, M. Loomis, J. Ryan, K. Bennett, and C. Winkler

Citation: AIP Conference Proceedings 294, 100 (1994); doi: 10.1063/1.45207

View online: http://dx.doi.org/10.1063/1.45207

View Table of Contents:

http://scitation.aip.org/content/aip/proceeding/aipcp/294?ver=pdfcov

Published by the AIP Publishing

Articles you may be interested in

OSSE observations of the 4 June 1991 solar flare

AIP Conf. Proc. 294, 15 (1994); 10.1063/1.45214

EGRET observations of extended highenergy emissions from the nuclear line flares of June 1991

AIP Conf. Proc. 294, 94 (1994); 10.1063/1.45206

Neutron and gammaray measurements of the solar flare of 1991 June 9 AIP Conf. Proc. 294, 89 (1994); 10.1063/1.45205

COMPTEL observations of gammaray flares in October 1991

AIP Conf. Proc. 294, 55 (1994); 10.1063/1.45200

COMPTEL's solar flare catalog

AIP Conf. Proc. 294, 51 (1994); 10.1063/1.45199 


\title{
Observations of the 1991 June 11 solar flare with COMPTEL
}

\author{
G. Rank, R. Diehl, G. G. Lichti, V. Schönfelder, M. Varendorff* \\ Max-Planck Institut für Extraterrestrische Physik, D-8046 Garching, Germany
}

B. N. Swanenburg, R. van Dijk**

SRON-Leiden, P.B. 9504, NL-2300 RA Leiden, The Netherlands

D. Forrest, J. Macri, M. McConnell, M. Loomis, J. Ryan

University of New Hampshire, Institute for the Study of Earth, Oceans and Space,

Durham NH 03824, U.S.A.

K. Bennett, C. Winkler

Astrophysics Division, Space Science Department of ESA/ESTEC, NL-2200

AG Noordwijk, The Netherlands

\begin{abstract}
The COMPTEL instrument onboard the Compton Gamma-Ray Observatory (CGRO) is sensitive to $\gamma$-rays in the energy range from 0.75 to $30 \mathrm{MeV}$ and to neutrons in the energy range from 10 to $100 \mathrm{MeV}$.

During the period of unexpectedly high solar activity in June 1991, several flares from active region 6659 were observed by COMPTEL. For the flare on June 11, we have analyzed the COMPTEL telescope data, finding strong $2.223 \mathrm{MeV}$ line emission, that declines with a time constant of 11.8 minutes during the satellite orbit in which the flare occurs. It remains visible for at least 4 hours. We obtained preliminary values for the $2.2 \mathrm{MeV}$ and 4-7 MeV fluences. Neutrons with energies above $20 \mathrm{MeV}$ have been detected and their arrival time at the Earth is consistent with the $\gamma$-ray emission during the impulsive phase.
\end{abstract}

\section{INTRODUCTION}

COMPTEL is a useful instrument for studying solar flares. It is able to measure $\gamma$ -rays in the energy range from $0.75 \mathrm{MeV}$ to $30 \mathrm{MeV}$ and to derive the direction of the incoming radiation. When operating in its full-telescope mode, COMPTEL uses a double scattering of a $\gamma$-quantum to get spectral and directional information. In this case, Compton scattering of the $\gamma$-ray is the fundamental process.

\footnotetext{
* Present adress: University of New Hampshire, Institute for the Study of Earth, Oceans and Space, Durham NH 03824, U.S.A.

**Also: Astronomical Institute "Anton Pannekoek", University of Amsterdam, The Netherlands
} 
In addition, COMPTEL has the unique capability of detecting individual neutrons in the energy range from approximately $10 \mathrm{MeV}$ to $100 \mathrm{MeV}$. The measurement is similar to that of $\gamma$-rays, but for neutrons hard-sphere scattering is the basic mechanism. The COMPTEL data undergo a pre-selection onboard the satellite, where a possible neutron signal is separated, mainly by a discrimination in pulse shape, and telemetered in a special neutron event stream (for details see Ryan et al., 1992; McConnell et al., these proceedings).

To avoid background of charged particles, the whole instrument is shielded by anti-coincidence plastic scintillators. To suppress additional $\gamma$-ray and neutron background originating in the Earth's atmosphere and the instrument itself, only events being compatible with the direction of the Sun are accepted. This uses the imaging capability of COMPTEL, as it has been demonstrated earlier for the June 11 solar flare (Rank et al., 1992).

The remaining background shows orbital variations due to changes in rigidity and spacecraft orientation relative to the Earth. However, 15 and 16 orbits before and after the flare, the orbital parameters are reproduced quite precisely. Therefore, these data can be used to generate a model background.

The interpretation of the data is very difficult due to several effects which are caused by the enormous $\mathrm{X}$ - and $\gamma$-ray flux during the flare: There are (1) severe deadtime effects, (2) the occurence of multi-hit processes in the telescope and (3) an overflow of the limited event buffers and the telemetry capability.

\section{OBSERVATIONAL RESULTS}

On June 11, 1991, the active solar region 6659 has produced a huge flare, being accompanied by a prominence. Optically it was classified as a 3B event and in soft Xrays as a X-12 GOES event. X-ray emission started at 1:56 UT, reached its maximum at 2:09 UT and faded away at about 2:20 UT. Orbital sunrise of CGRO had taken place at 1:48 UT, a few minutes before the flare onset. Hence, the temporal evolution of the flare could be studied for a whole orbital period.

\section{Analysis of COMPTEL $\gamma$-ray measurements:}

A time history of the June 11 solar flare can be obtained from COMPTEL telescope data by correcting the measured count rate for background and live-time effects. The $\gamma$-ray emission starts at about 1:58 UT, shows a first peak at 2:00 UT and reaches its maximum at 2:05 UT. This indicates a faster rise to the maximum than in soft Xrays. After the maximum, the $\gamma$-ray emission in the $2.0-2.5 \mathrm{MeV}$ range shows exponential decline with a time constant of 11.8 minutes during the flare orbit. In the next two orbits a line signal is still remaining. This indicates the production of the 2.223 $\mathrm{MeV}$ line and therefore the presence of neutrons for at least 4 hours after the impulsive phase (McConnell et al., 1992; Rank et al., 1992).

To obtain preliminary values for fluences, we set tight restrictions on the incoming direction of the $\gamma$-rays in order to get a photo-peak response for the detectors. In this case, the effective areas from the calibration can be used. They have to be corrected for data restrictions (some restrictions are different from the calibration standards) and for the incomplete module combination (two modules of the lower de- 


\section{Observations of the 1991 June 11 Solar Flare with COMPTEL}

tector were turned off). The remaining effective areas are in the order of $6 \mathrm{~cm}^{2}$, slightly depending on energy.

The fluences were calculated for the whole flare orbit, beginning at the onset of the flare (2:00 UT) and ending at the orbital sunset (3:00 UT). For the 4-7 MeV range we obtain a fluence of $\phi_{4-7}=138 \pm 8 \gamma \mathrm{cm}^{-2}$. For the $2.223 \mathrm{MeV}$ line the fluence in the range from 2.1 to $2.4 \mathrm{MeV}$ is given by $\phi_{2.2}=114 \pm 18 \gamma \mathrm{cm}^{-2}$. This leads to a fluence ratio of $\phi_{2.2} / \phi_{4-7}=0.83 \pm 0.14$.

Assuming the same acceleration conditions as they have been studied by Hua and Lingenfelter (1987), a preliminary estimate of the spectral hardness of the accelerated proton spectrum can be made. In the case of a power law spectrum, as it would appear for non-relativistic shock acceleration, we get a spectral index of $s=3.6$. For second order stochastic acceleration we would expect a Bessel function with $\alpha \mathrm{T}=0.02$.

Analysis of COMPTEL neutron measurements:

The analysis of the neutron data is performed in the same way as it has been done for the $\gamma$-rays. However, some effects complicate the analysis:

During the most intense part of the flare, pulse pile-up effects of the $\gamma$ - and X-ray flux produce a strong signal which is not related to incoming neutrons. These events are arriving at the Earth when the electromagnetic signal is expected. But calculating their emission time at the Sun, they are emitted long before the flare onset. Data restrictions on the energy thresholds of the detectors, combined with tighter windows on the pulse shape discrimination and the time of flight from the upper to the lower detector remove the bulk of the artifical counts during the impulsive phase. However, a few counts still remain. These are scaled up by huge live time factors producing single spikes before the onset of the flare. As they are not related to neutrons they were excluded in the figures.

A phase of extremely low count rate follows this artificial signal. It is caused by the dead-time effects due to the huge $\mathrm{X}$ - and $\gamma$-ray flux. The dead-time situation is improving when the neutrons arrive. Nevertheless, a live-time correction has been applied to the neutrons in the same manner as was done for the $\gamma$-rays.

In figures 1 to 4 the time histories of the neutrons are displayed. The count rates in the four energy ranges $10-20 \mathrm{MeV}, 20-40 \mathrm{MeV}, 40-60 \mathrm{MeV}$ and $60-100 \mathrm{MeV}$ are calculated back to their emission time at the Sun. A phase of negative values appears in each picture. It is caused by a suppression of counts beneath the background level during the impulsive phase when the dead time is great. The start and end time of each plot is defined by the satellite orbit.

In the lowest energy range almost no signal can be found in the flare orbit. Neutrons with these energies are travelling so slow that they arrive at the end of the satellite orbit when the neutron background from the Earth's atmosphere makes a detection difficult (most recent analysis performed after the Workshop has shown that there is an excess during the next two satellite orbits. This would be in very good agreement with the extended signal of the $2.223 \mathrm{MeV}$ line).

For the higher energy ranges, especially for the 40-60 MeV range, a significant signal can be found. The events are measured at times when neutrons from the impulsive phase are expected to reach the Earth. 


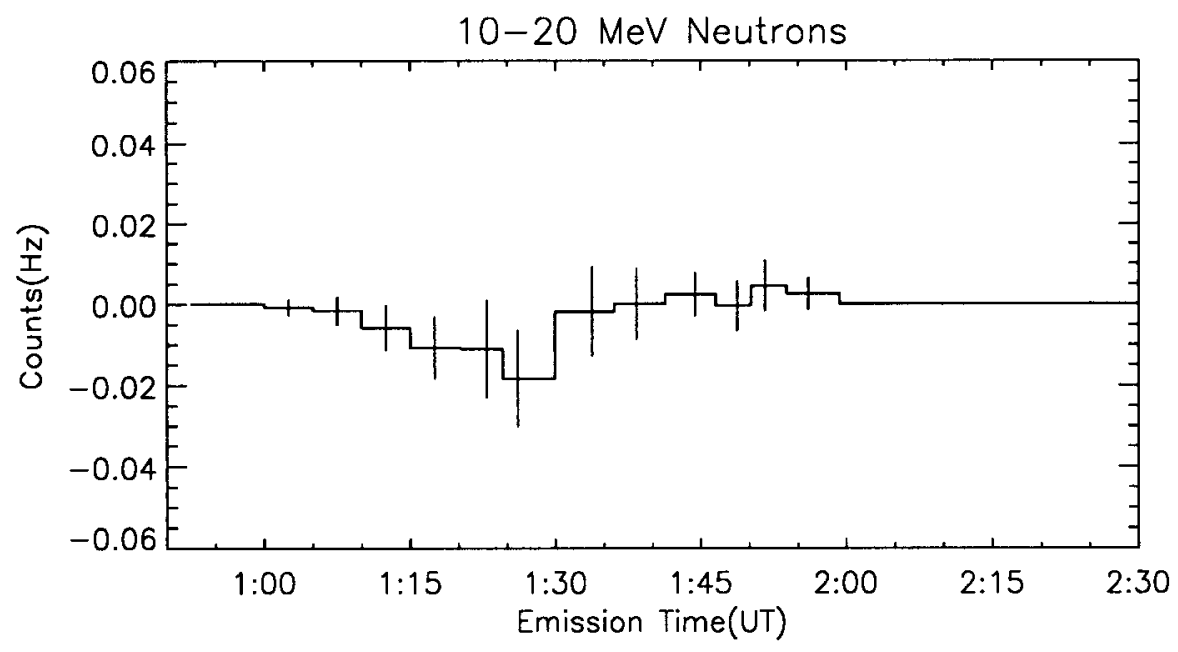

Fig. 1: Time history of the count rate of neutrons in the energy range from $10 \mathrm{MeV}$ to $20 \mathrm{MeV}$ for their calculated emission time at the Sun. The $\gamma$-ray event at the sun peaks at about 1:55 UT.

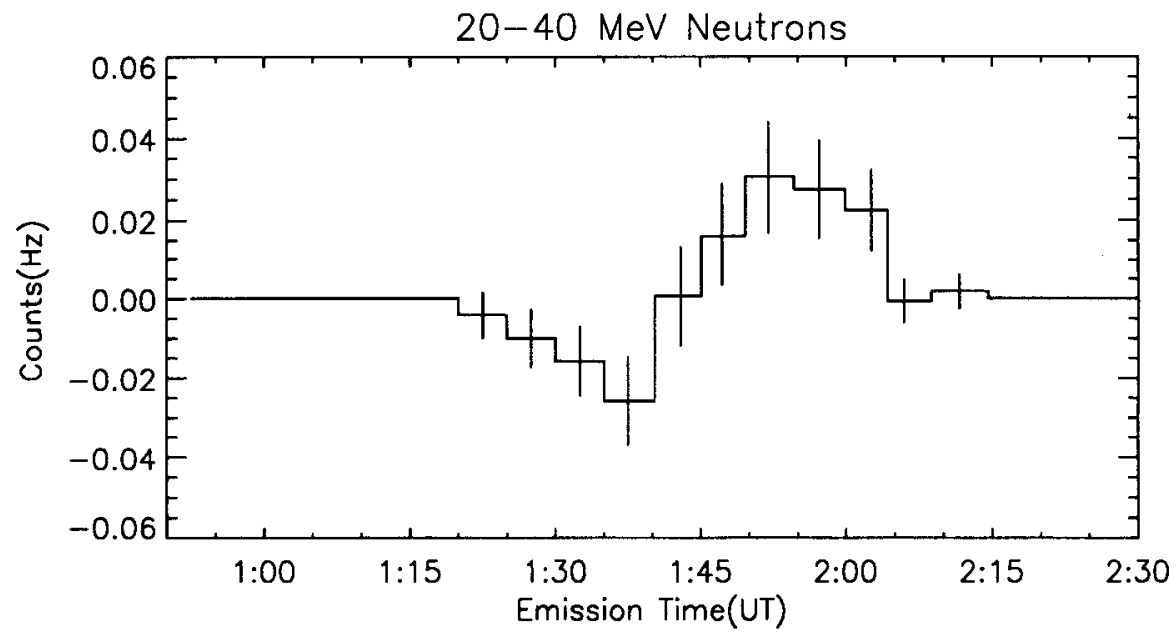

Fig. 2: Time history for neutrons in the energy range from $20 \mathrm{MeV}$ to $40 \mathrm{MeV}$ (see also Fig. 1). 
104 Observations of the 1991 June 11 Solar Flare with COMPTEL

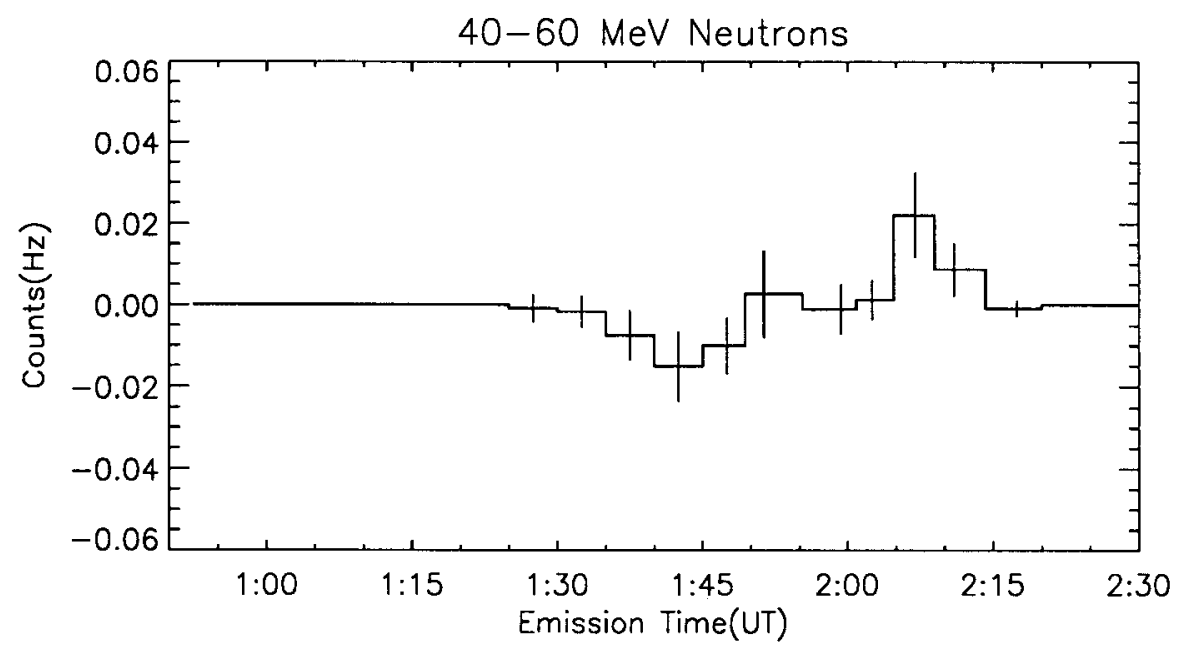

Fig. 3: Time history for neutrons in the energy range from $40 \mathrm{MeV}$ to $60 \mathrm{MeV}$ (see also Fig. 1).

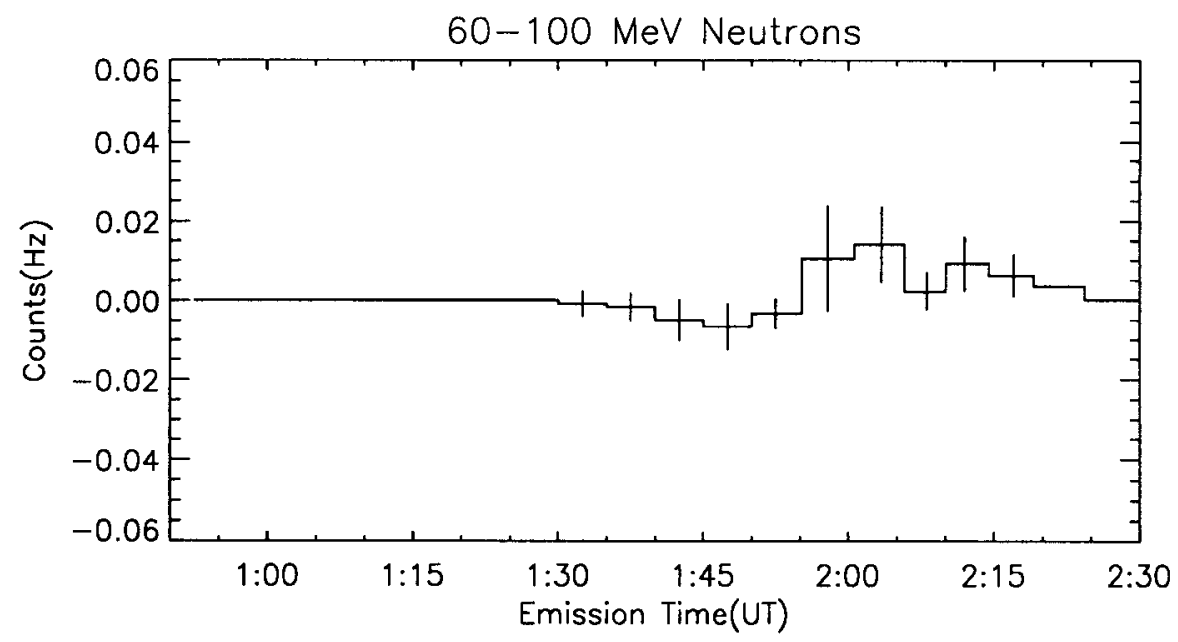

Fig. 4: Time history for neutrons in the energy range from $60 \mathrm{MeV}$ to $100 \mathrm{MeV}$ (see also Fig. 1). 


\section{SUMMARY}

The spectrum of the 1991 June $11^{\text {th }}$ flare shows a strong $2.223 \mathrm{MeV}$ line that can be seen by COMPTEL for at least 4 hours after the impulsive phase.

Neutrons in the energy range from approximately 20 to $100 \mathrm{MeV}$ can be detected during the flare orbit. Their calculated emission-time profile at the Sun matches well with the impulsive phase as defined by the $\gamma$-rays.

\section{REFERENCES}

X. M. Hua, R. E. Lingenfelter, Solar Physics, 107, 351-383 (1987).

M. McConnell, K. Bennett, H. Bloemen, H. de Boer, M. Busetta, W. Collmar, A. Connors, R. Diehl, J.W. den Herder, W. Hermsen, L. Kuiper, G. G. Lichti, J. Lockwood, J. Macri, D. Morris, R. Much, G. Rank, J. Ryan, V. Schönfelder, G. Stacy, H. Steinle, A. W. Strong, B. N. Swanenburg, B. G. Taylor, M. Varendorff, C. de Vries, W. Webber, C. Winkler, COMPTEL observations of solar flare gamma-rays (COSPAR, 1992).

M. McConnell, K. Bennett, H. Bloemen, H. Debrunner, R. Diehl, D. Forrest, L. Hanlon, W. Hermsen, G. G. Lichti, J. Lockwood, M. Loomis, G. Rank, J. Ryan, V. Schönfelder, A. W. Strong, B. N. Swanenburg, M. Varendorff, C. Winkler, An Overview of Solar Flare Results from COMPTEL, (High Energy Physics of Solar Flares, 1993).

G. Rank, K. Bennett, R. Diehl, D. Forrest, L. Hanlon, G. G. Lichti, J. Macri, M. McConnell, J. Ryan, V. Schönfelder, B. N. Swanenburg, M. Varendorff, C. Winkler, Observations of the 1991 June 11 solar flare with COMPTEL (Compton Symposium, 1992).

J. Ryan, H. Aarts, K. Bennett, R. Byrd, C. de Vries, J. W. den Herder, A. Deerenberg, R. Diehl, G. Eymann, D. Forrest, C. Foster, W. Hermsen, G. G. Lichti, J. Lockwood, J. Macri, M McConnell, D. Morris, V. Schönfelder, G. Simpson, M. Snelling, H. Steinle, A. W. Strong, B. N. Swanenburg, T. Taddeucci, W. R. Webber, C. Winkler, COMPTEL as a solar gamma ray and neutron detector (Data Analysis in Astronomy IV, ed. V.D.Gesú et al., 1992). 